\title{
Physiological differences between burnout patients and healthy controls: blood pressure, heart rate, and cortisol responses
}

\author{
W De Vente, M Olff, J G C Van Amsterdam, J H Kamphuis, P M G Emmelkamp
}

Occup Environ Med 2003;60(Suppl I):i54-i61

See end of article for
authors' affiliations
$\ldots \ldots \ldots \ldots \ldots \ldots \ldots \ldots \ldots$
Correspondence to:
W De Vente, Department
of Clinical Psychology,
University of Amsterdam,
Roetersstraat 15, 1018
WB Amsterdam, The
Netherlands;
devente@psy.uva.nl
Accepted
1 March 2003

\begin{abstract}
Objectives: To investigate differences between burnout patients and healthy controls regarding basal physiological values and physiological stress responses. Measures of the sympathetic-adrenergicmedullary (SAM) axis and the hypothalamic-pituitary-adrenal (HPA) axis were examined.

Methods: SAM axis and HPA axis activity was compared between 22 burnout patients and 23 healthy controls. SAM axis activity was measured by means of heart rate (HR) and blood pressure (BP). HPA axis activity was investigated by means of salivary cortisol levels. Resting levels of HR, BP, and cortisol were determined as well as reactivity and recovery of these measures during a laboratory session involving mental arithmetic and speech tasks. In addition, morning levels of cortisol were determined. Results: Burnout patients showed higher resting HR than healthy controls. BP resting values did not differ between burnout patients and healthy controls, nor did cardiovascular reactivity and recovery measurements during the laboratory session. Basal cortisol levels and cortisol reactivity and recovery measures were similar for burnout patients and healthy controls. However, burnout patients showed elevated cortisol levels during the first hour after awakening in comparison to healthy controls.

Conclusions: The findings provided limited proof that SAM axis and HPA axis are disturbed among burnout patients. Elevated HR and elevated early morning cortisol levels may be indicative of sustained activation.
\end{abstract}

A though originally exclusively related to social professions, burnout is nowadays commonly accepted as a stress state characterised by symptoms such as mental exhaustion and physical fatigue, detachment from work, diminished competence, loss of energy, increased irritability and sleep, and concentration problems which can occur irrespective of the type of profession. ${ }^{1}$ The symptoms of burnout are also known as subjective health complaints, as up until now, no objective signs of illness have been found for these complaints. Comparable symptom clusters, with exclusion of the work related complaints, ${ }^{2}$ are also characteristic of chronic fatigue syndrome (CFS) ${ }^{3}$ and post traumatic stress disorder (PTSD). ${ }^{4}$ Although burnout is not considered to be a "classical" stress disorder, it is often preceded by periods of prolonged work related stress. ${ }^{1}$

Concerning the relation between stress and health complaints, attention is paid within psychobiological research to the association between failure to effectively cope with stressors and physiological responses. ${ }^{5}$ Two physiological stress systems are commonly distinguished: the sympatheticadrenergic-medullary (SAM) axis and the hypothalamicpituitary-adrenal (HPA) axis..$^{5-7}$ Simply stated, the SAM axis concerns immediate sympathetic activation preparing an individual to deal with a stressor, resulting in for example increased heart rate (HR) and blood pressure (BP) and release of catecholamines such as epinephrine and norepinephrine. SAM activation occurs within seconds as a result of a stressor and permits adaptive responding to a stressor. The HPA axis is a slower response system involving release of corticosteroids such as corticotropin releasing hormone, adrenocorticotropic hormone, and cortisol, resulting in immune suppression. HPA activation is associated with inability to cope, helplessness, affective distress, and perceived uncontrollability. ${ }^{5-8}$ Generally, low SAM and HPA activation is seen in persons with high levels of effective coping and control.9-11

According to the "sustained activation theory", sustained or frequently repeated activation of the stress systems without the possibility to rest resulting in chronic allostatic load, may develop in illness. ${ }^{2}$ Sustained activation can be a result of pro- longed or repeated exposure to stressors, with which a person cannot adequately cope. Consequently, the SAM axis and HPA axis stay persistently activated, recovery does not occur, and the systems do not return to homeostasis. Lasting changes in the neuroendocrine system are thought to be particularly health threatening. ${ }^{12-14}$

Elevated BP or hypertension may be considered a sign of dysregulation of the SAM axis while hypersecretion or hyposecretion of cortisol is indicative of dysregulation of the HPA axis. Associations between SAM and HPA functioning on the one hand, and workload, job strain, and chronic stress on the other, have been repeatedly observed. Elevations in $\mathrm{BP}^{15-18}$ and $\mathrm{HR}^{15}{ }^{16}$ during the workday were shown in various populations and professions. In addition, positive associations were found between job strain and $\mathrm{BP}^{19-21}$ job strain and hypertension, ${ }^{22}$ and between chronic stress and cardiovascular basal levels. ${ }^{23}$ Furthermore, relations were observed between cortisol and workload or job strain. ${ }^{24} 25$

Since burnout symptoms highly resemble symptoms that are characteristic of CFS, Frankenhaeuser ${ }^{26}$ suggested that burnout patients as $\mathrm{CFS}^{27}{ }^{28}$ patients might show lower basal HPA axis activity resulting for example in hyposecretion of cortisol. Basal hyposecretion of cortisol has also been observed in individuals who developed PTSD after an extreme stressor compared to normal subjects and other psychiatric patients. ${ }^{29-31}$ However, studies concerning chronic stress due to high job strain, work overload, and/or vital exhaustion show both decreases ${ }^{32}{ }^{33}$ and increases ${ }^{25}{ }^{34-36}$ in cortisol levels. Results from the study of Melamed et $a l^{35}$ suggested that within a

Abbreviations: BDI, Beck Depression Inventory; BP, blood pressure; CFS, chronic fatigue syndrome; CIDI, Composite International Diagnostic Interview; $\mathrm{CIS}$, checklist individual strength; HPA,

hypothalamic-pituitary-adrenal; HR, heart rate; $M A$, mental arithmetic; POMS, Profile of Mood Scale; PTSD, post traumatic stress disorder; REC 1, first recovery phase; REC2, second recovery phase; SAM, sympathetic-adrenergic-medullary 
sample of employees, a longer history of burnout complaints implying chronicity was associated with higher cortisol levels.

With respect to the relationship between stress and health, it appears relevant to investigate reactivity to and recovery from stressors, rather than looking at basal values of SAM activation and HPA activation alone. ${ }^{5}$ Different reactivity might occur concerning the magnitude and/or the duration of the response. Likewise, recovery may not come about to the same extent and/or within the same time frame. McEwen ${ }^{37}$ has stated that delayed recovery following a stressor is indicative of sustained activation. Kristenson et $a l^{34}$ found that elevated cortisol levels among persons reporting job strain and vital exhaustion were associated with attenuated cortisol reactivity to stress inducing tasks. Roy et $a^{38}$ found that among healthy fire fighters reporting a higher density of recent life events, high social support was associated with stronger HR and BP reactivity, and quicker recovery to stress inducing tasks in comparison with low social support. According to Roy et $a l^{38}$ swifter recovery despite increased reactivity may be seen as adequate adaptation.

It should be noted that above mentioned studies were carried out among participants who could still carry out their daily activities. Thus, although participants reported chronic stress or even burnout symptoms, these conditions were not impairing their daily functioning to a large extent. To our knowledge, studies concerning SAM axis and HPA axis functioning among burnout patients who are unable to work are absent.

The objective of the present study is to investigate differences between burnout patients and healthy controls concerning SAM axis and HPA axis functioning as indicated by both basal values and reactivity and recovery measures. Therefore, burnout patients and healthy controls visited the laboratory to perform mental arithmetic and speech tasks. In addition, participants collected morning saliva to obtain information about the cortisol response to awakening, which, according to Pruessner $e t \mathrm{al}^{39}$ shows good intra-individual stability over time and can serve as an indicator for HPA axis activity. We expected to find dysregulation of SAM axis and HPA axis functioning in burnout patients.

\section{METHODS}

\section{Participants}

Forty-five participants took part in the present study, consisting of 22 burnout patients and 23 healthy persons. Group characteristics such as gender distribution, average age, education level, and type of occupation are presented in table 1. Healthy controls were younger than burnout patients $(\mathrm{t}(43)=4.0, \mathrm{p}<0.001)$.

Burnout patients were included in the study if (1) they reported stress complaints such as emotional and physical fatigue, loss of energy, increased irritability, and sleep and concentration problems; (2) the complaints were considered to be predominantly work related; and (3) participants reported burnout related (partial) sick leave, which has lasted between two weeks to three months full time, or six months part time. A semi-structured diagnostic interview was administered by a clinical psychologist. Primary Axis I disorders were excluded by a short version of the Composite International Diagnostic Interview (CIDI), ${ }^{40}$ a structured interview covering depression, social phobia, panic disorder, somatoform disorder, post-traumatic stress disorder, obsessive-compulsive disorder, hypomania, and psychotic disorders. Additionally, the Beck depression inventory (BDI) ${ }^{41}$ was used to exclude severe depression. Since depressive symptoms such as difficulty concentrating and loss of energy are also characteristic of burnout, the cut off score was set at a conservative 25 .

Healthy individuals were screened by a telephone interview. Participants in good physical health and working for at least eight hours a week were included in the study. Exclusion criteria were: having experienced a traumatic event in the past six months, psychiatric illness, pregnancy, currently taking sick leave and scoring both within the clinical range of the mental exhaustion scale of the Maslach burnout inventory $(>2.60)^{42}$ and within the clinical range of the total fatigue score of checklist individual strength $(>76) .^{43}$

Participants reporting a history of immune, diabetic, or other medical disease that could possibly explain fatigue were excluded from the study. Two burnout patients were using anti-hypertensive medication in the form of beta-blockers. No healthy controls were using medication, except for three women who were on oral contraceptives.

Burnout patients were recruited through occupational health practitioners and general practitioners. Healthy subjects were recruited by flyers in libraries and other public places, and among part time working psychology students. Healthy subjects were paid $€ 20$ after attending the laboratory session, completion of the questionnaires, and delivering the morning saliva samples. Burnout patients received refund of their travel expenses. Burnout patients and healthy controls received a printed report of their baseline BP and HR. All participants in the study gave written informed consent.

\section{Procedure}

Participants collected morning saliva, visited the laboratory in order to perform mental arithmetic and speech tasks, and filled out questionnaires concerning biographic characteristics, burnout, and fatigue. On the day of morning saliva collection and on the day of stress test performance, participants filled out a state questionnaire pertaining to mood, physical activity, and smoking. Participants were asked to refrain from smoking and coffee consumption for at least 60 minutes before the start of the laboratory session. The stress test was held in the afternoon, starting at 13.30, in order to reliably differentiate the experimentally induced cortisol from the strong early morning elevation, which is inherent to the circadian rhythm of the morning cortisol secretion. ${ }^{44}$

To prevent effects of anticipatory stress for the laboratory session on morning cortisol levels, morning saliva was collected on another day than the laboratory session. With the exception of five participants, all burnout patients collected the morning saliva on the day before they visited the laboratory. The control group collected the morning saliva

\begin{tabular}{|c|c|c|}
\hline & $\begin{array}{l}\text { Burnout patients } \\
(\mathrm{n}=22)\end{array}$ & $\begin{array}{l}\text { Healthy controls } \\
(n=23)\end{array}$ \\
\hline \multicolumn{3}{|l|}{ Gender } \\
\hline Men & $14(64 \%)$ & $10(43 \%)$ \\
\hline Women & $8(36 \%)$ & 13 (57\%) \\
\hline Age & $42(10.2)$ & $31(7.6)^{c}$ \\
\hline Cigarette smokers & $9(41 \%)$ & $7(30 \%)$ \\
\hline \multicolumn{3}{|l|}{ Burnout (UBOS) } \\
\hline Mental exhaustion & $4.2(1.0)$ & $1.3(0.8) \ddagger$ \\
\hline Distance & $3.1(1.2)$ & $1.4(0.9) \ddagger$ \\
\hline Competence & $4.1(1.0)$ & $4.3(1.0)$ \\
\hline Fatigue (CIS total score) & 105.4 (19.7) & $47.8(22.8) \ddagger$ \\
\hline \multicolumn{3}{|l|}{ Type of occupationt } \\
\hline Non-social & $11(48 \%)$ & $15(62 \%)$ \\
\hline Social & $12(52 \%)$ & $9(38 \%)$ \\
\hline \multicolumn{3}{|l|}{ Duration of complaints } \\
\hline$<3$ months & $1(4 \%)$ & - \\
\hline$>3$ and $<6$ months & $3(14 \%)$ & - \\
\hline$>6$ and $<12$ months & $10(46 \%)$ & - \\
\hline$>12$ months & $8(36 \%)$ & - \\
\hline
\end{tabular}

*Values are counts (percentages) and means (SD).

†Social occupation includes human service occupations, teachers, and managers.

$\ddagger$ Difference with patient group is significant at alpha $<0.05$. 
within five days after their visit to the laboratory. Instructing participants to collect morning saliva on a weekday avoided disturbing influences due to different sleeping habits at the weekend.

\section{Laboratory session}

In the laboratory session an adapted version of the Trier Social Stress Test ${ }^{46}$ was administered, consisting of (a) an adaptation period (30 mins), (b) speech task preparation ( 5 mins), (c) a mental arithmetic task ( 5 mins), (d) a speech task ( 5 mins) followed by (e) two resting/recovery periods ( 15 mins each). In the adaptation period the participant was introduced to the procedures and the blood pressure instrumentation. Furthermore, the participant watched a documentary-like movie for 15 minutes. In the speech task preparation period the participant was instructed to read a short description of a situation in which they were unfairly accused of causing damage. The participant was told to imagine themself in that particular situation and to think how they would react behaviourally, emotionally, etc. The participant was also told to prepare a consistent story, which they had to tell in front of a camera later that afternoon. The mental arithmetic task entailed adding and subtracting rows of five to seven integers while only writing down the final solution per row. The participant was instructed to solve the sums as quickly as possible, without making mistakes. For the speech task, the participant was told to tell their story which had to last five minutes. If the participant silenced before the five minutes had passed, they were told to keep talking and if they silenced a second time, the experimenter reported how much time there was left. In the following resting periods, participants again watched the subsequent part of the documentary-like movie. During the whole experimental session, participants remained seated. This procedure has been shown to induce subjective stress as well as changes in endocrine and immune parameters. ${ }^{47}$

\section{Cardiovascular measures}

Heart rate and blood pressure were computed from continuous finger blood pressure using a Finapres blood pressure monitir (Ohmeda Finapress type $2300^{\mathrm{E}}$ ). Baseline values were obtained by calculating average values during the final ten minutes of the adaptation period. Task reactivity was determined by calculating average values over each task (preparation, mental arithmetic, speech task) separately. Short and long term recovery were measured by averaging values over the first and second resting periods respectively, each lasting 10 minutes (with exclusion of saliva collection).

\section{Saliva collection and cortisol assay}

During the laboratory session, saliva was collected five times: (1) at baseline ( $\mathrm{t}=-4$ mins); (2) immediately after the preparation phase ( $\mathrm{t}=5$ mins); ( 3 ) directly after the speech task $(\mathrm{t}=19$ mins); (4) after the first resting period ( $\mathrm{t}=33 \mathrm{mins})$; and (5) after the second resting period $(t=47)$. Saliva collection was conducted as indicated by Navazesh..$^{48}$ Following this method, the participant refrains from swallowing for a period of four minutes, allowing the saliva to accumulate in the floor of the mouth. The participant spits out into a cup every 60 seconds. Fifteen minutes before the experiment, the participant rinses the mouth with water. The collection starts with the instruction to void the mouth of saliva by swallowing. After collection, saliva samples were stored upon ice until the end of the experiment. Immediately after the experiment, saliva was clarified by centrifugation ( $10000 \mathrm{~g}$, $4 \mathrm{mins}$ ). The clear supernatant was divided in $0.5 \mathrm{ml}$ aliquots and stored at $-20^{\circ} \mathrm{C}$ until analysis.

With respect to morning cortisol levels, participants were instructed to collect saliva at the time of awakening, 30 minutes, and 60 minutes thereafter and at 12.00 hours (am). Saliva was collected in non-coated Salivettes ${ }^{\mathrm{TM}}$ (Sarstedt,
Nümbrecht, Germany) placed under the tongue or between cheek and teeth for three minutes. If the swab was not saturated, subjects were permitted to slowly move the swab around in the mouth without chewing on it. Participants were instructed not to have breakfast or brush their teeth within 15 minutes before a sample was collected. Additionally, participants were instructed to store all samples in the fridge until next day's visit to the laboratory (burnout patients) or postage/deliverance (healthy controls). All samples were centrifuged at $(5000 \mathrm{~g}, 5 \mathrm{mins})$ and stored at $-20^{\circ} \mathrm{C}$ until analysis. The amount of free cortisol was determined by enzyme immunoassay (DSL, Veghel, The Netherlands). Sensitivity of assay was $1 \mathrm{ng} / \mathrm{ml}$.

\section{Psychological variables}

The Dutch version of the Maslach Burnout Inventory-General Survey (MBI-GS) was used to measure burnout (Utrechtse Burnout Schaal: UBOS). ${ }^{42}$ The UBOS consists of 15 items regarding mental exhaustion, distance, and competence, which are scored on a seven point Likert scale ranging from 1 (never) to 7 (always/daily).

General fatigue was measured with the Checklist Individual Strength (CIS). ${ }^{49}$ The CIS consists of 20 items referring to four dimensions of fatigue: (a) subjective feeling of fatigue and physical fitness, (b) activity level, (c) motivation, and (d) concentration, which are scored on a seven point Likert scale ranging from 1 (false) to 7 (true). A total fatigue score is calculated by adding all item scores and can thus range from 20 to 140 .

The subjective response during the laboratory session was measured by means of the Vigor and Tension subscales of the Profile Of Mood Scale (POMS), consisting of five and six items respectively. ${ }^{50}$ Items are scored on a five point scale ranging from 0 (not at all) to 4 (very much). The Vigor subscale is indicative of psychological activation and the Tension subscale contains items referring to tension and anxiety. These mood dimensions have been previously used to measure subjective response during stress inducing tasks. ${ }^{51}$ The mood questionnaires were completed five times during the laboratory session, while saliva was collected.

\section{Occupation and education}

Type of occupation was categorised according to the social character of the profession. Two categories were distinguished: (1) human service occupations, teachers and managers; (2) less/non-social occupations-for example, writer, truck driver, administrative employee). Average education level has been assessed as the highest completed education on a six point scale ranging from 1 (primary school) to 6 (university grade).

\section{STATISTICAL ANALYSES}

Proportion of smokers, type of profession, mental exhaustion, and fatigue were compared between the patient group and the healthy controls using $\chi^{2}$ tests and $t$ tests. Group differences in resting values of cardiovascular measures during the adaptation phase and the second resting phase of the laboratory session were analysed by means of analysis of variance (ANOVA), using one between-subjects (group) design. Basal cortisol levels measured on midday (fourth sample of the morning saliva samples) and at 15.00 (final sample of the laboratory session) were analysed in the same manner (ANOVA, using group as one between-subjects factor).

Reactivity and recovery during the laboratory session were analysed by means of ANOVA for repeated measures, using a one within- (time), one between-subjects (group) design for the cardiovascular measures, the cortisol outcomes, and the subjective responses separately. Early morning cortisol response was analysed likewise. If the assumption of sphericity was violated, corrected results according to GreenhouseGeisser method were presented. Post hoc contrasts were 
employed to reveal group differences concerning changes between subsequent measurements. Analyses were performed with and without relevant covariates. Both age and gender have consistently been associated with cardiovascular measures and cortisol levels ${ }^{22}{ }^{52-55}$ and were therefore added as covariates, despite the fact that the difference of gender distribution was not statistically significant between the burnout patients and healthy controls.

Some data were missing due to equipment problems (eg unrecorded data) or to lost saliva samples. Analysis of blood pressure and heart rate was based on 22 burnout patients and 22 healthy controls, with the exception of baseline values during the adaptation period, which was based on 23 healthy controls. In addition, analysis of cortisol during the laboratory session was based on 21 burnout patients and 23 healthy controls, as was comparison of baseline values at 15.00 hours. Furthermore, analysis of morning cortisol was carried out for 20 burnout patients and 23 healthy controls, with exception of baseline comparison at 12.00 hours, which was based on 21 burnout patients and 23 healthy controls.

\section{RESULTS}

As expected, burnout patients scored significantly higher than healthy controls on the mental exhaustion subscale and the distance subscale of the UBOS $(\mathrm{t}(43)=11.0, \mathrm{p}<0.001 ; \mathrm{t}(43)$ $=5.3, \mathrm{p}<0.001)$ and on the CIS $(\mathrm{t}(43)=9.1, \mathrm{p}<0.001)$ ( see table 1). Within the group of burnout patients, the majority $(82 \%)$ reported chronic burnout complaints, that is complaints of more than six months duration.

\section{Cardiovascular measures}

Burnout patients had higher resting HR than healthy controls as measured during the adaptation and the second recovery phase of the laboratory session $(\mathrm{F}(1,43)=7.17, \mathrm{p}<0.05$; $\mathrm{F}(1,42)=5.93, \mathrm{p}<0.05)$. A trend in the same direction was found for systolic BP during the second recovery phase $(\mathrm{F}(1,42)=3.14, \mathrm{p}<0.10)$, indicating higher resting systolic BP for burnout patients compared to healthy controls. After correction for age and gender, the difference for HR during the adaptation phase remained statistically significant $(\mathrm{F}(1,41)=$ $4.21, \mathrm{p}<0.05)$, the difference during the second recovery phase became marginally significant $(\mathrm{F}(1,40)=3.75, \mathrm{p}<$ 0.10 ) and the difference of systolic BP was no longer significant $(F(1,41)=0.81, p>0.05)$.

Systolic BP, diastolic BP, and HR were different during the six laboratory session phases indicating a development over time (within-subjects effect of time: $F(5,210)=35.72, p<$ $0.001 ; \mathrm{F}(5,210)=58.09 \mathrm{p}<0.001 ; \mathrm{F}(5,210)=46.23, \mathrm{p}<$ 0.001 , respectively). Burnout patients had higher HR during the entire laboratory session (between-subjects group effect: $\mathrm{F}(1,42)=6.66, \mathrm{p}<0.05)$. No between-group differences were observed with respect to development over time for any of the cardiovascular measures during the laboratory session (timegroup interaction: systolic BP: $\mathrm{F}(5,210)=1.99, \mathrm{p}>0.05$; diastolic BP: $\mathrm{F}(5,210)=1.04, \mathrm{p}>0.05 ; \mathrm{HR}: \mathrm{F}(5,210)=0.25$, $\mathrm{p}>0.05$ ). Post hoc analyses revealed that overall (irrespective of group) differences between subsequent phases of the laboratory session were all statistically significant, with exception of diastolic BP between the adaptation and preparation phase and HR between the first and second recovery phase. After correction for age and gender, the within-subjects time effect remained statistically significant for systolic and diastolic BP $(\mathrm{F}(5,200)=4.29, \mathrm{p}<0.01 ; \mathrm{F}(5,200)=4.34, \mathrm{p}<0.01)$, and became marginally significant for $\operatorname{HR}(\mathrm{F}(5,200)=2.30, \mathrm{p}<$ $0.10)$. The between-subjects group effect remained statistically significant for $\operatorname{HR}(\mathrm{F}(1,40)=4.12, \mathrm{p}<0.05)$. Again, no time group interaction effects were found. Figures 1 to 3 show average systolic and diastolic BP and HR values during the different phases of the laboratory session .

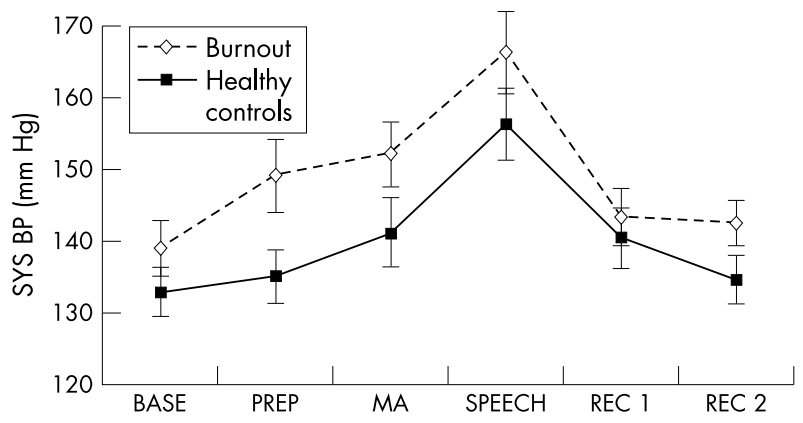

Figure 1 Mean systolic blood pressure (SEM) in burnout patients and healthy controls during six phases of the laboratory session. BASE, baseline; PREP, speech task preparation; MA, mental arithmetic; SPEECH, speech task; REC1, first recovery phase; REC2, second recovery phase.

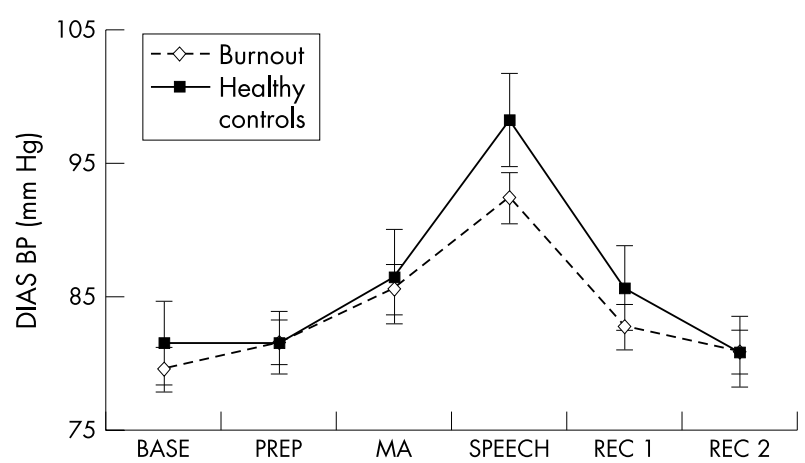

Figure 2 Mean diastolic blood pressure (SEM) in burnout patients and healthy controls during six phases of the laboratory session. BASE, baseline; PREP, speech task preparation; MA, mental arithmetic; SPEECH, speech task; REC1, first recovery phase; REC2, second recovery phase.

\section{Cortisol}

Basal values of cortisol measured at 12.00 hours and at 15.00 hours revealed no differences between burnout patients and healthy controls $(\mathrm{F}(\mathrm{l}, 42)=0.24, \mathrm{p}>0.05 ; \mathrm{F}(1,42)=0.18, \mathrm{p}$ $>0.05)$. Correction for age and gender did not change the results. During the laboratory session, cortisol changed over the different phases (within-subjects time effect: $\mathrm{F}(4,168)=$ $17.80, \mathrm{p}<0.001)$. No overall group differences emerged (between-subjects group effect: $F(1,42)=2.27, \mathrm{p}>0.05$ ). Burnout patients showed a different development of cortisol levels during the laboratory session in comparison to healthy

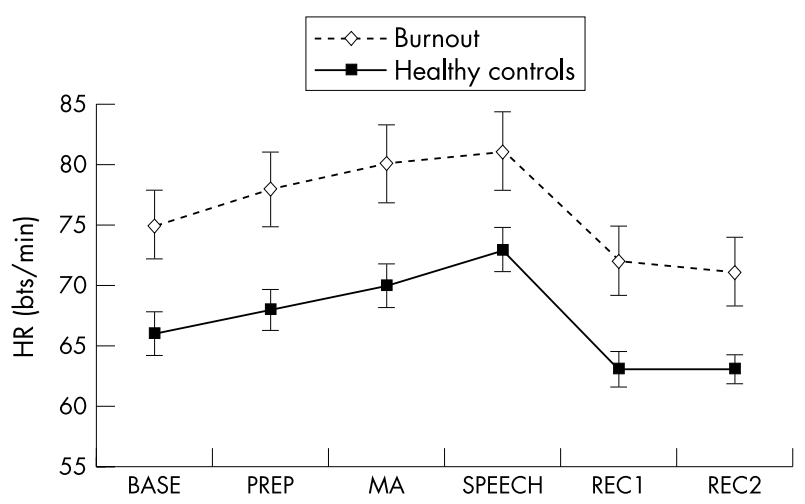

Figure 3 Mean heart rate (SEM) in burnout patients and healthy controls during six phases of the laboratory session. BASE, baseline; PREP, speech task preparation; MA, mental arithmetic; SPEECH, speech task; REC1, first recovery phase; REC2, second recovery phase. Statistically significant group difference (cross-sectionally) of HR resting values is indicated by enlargement of group indicators. 


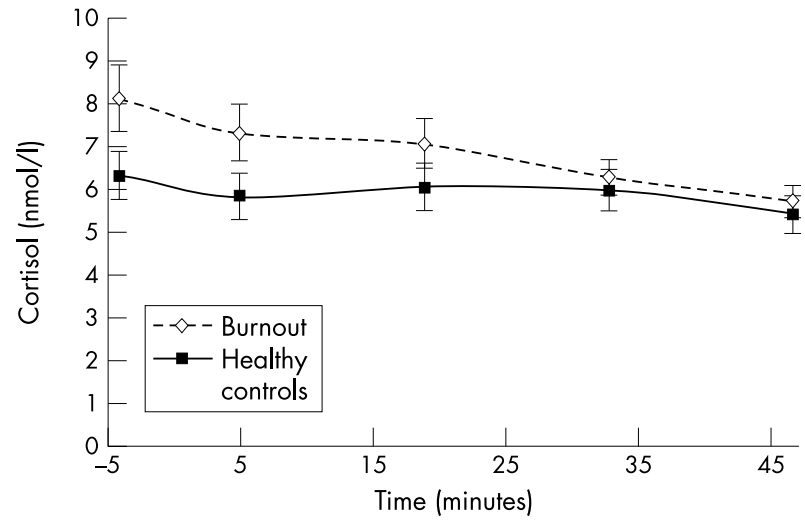

Figure 4 Mean concentration of salivary free cortisol (SEM) in burnout patients and healthy controls during the laboratory session (t $=-4$, baseline; $t=5$, post preparation sample; $t=19$, post speech sample; $t=33$, resting phase sample; $t=47$, recovery sample).

controls (time-group interaction effect: $F(4,168)=4.16, p<$ $0.05)$. Post hoc analyses of the overall within-subjects time effect revealed that differences between subsequent phases of the laboratory session were all statistically significant, with exception of the differences between the preparation and the speech task samples. The time group interaction effect could not be attributed to a particular phase, but is probably a result of the overall steeper decrease in cortisol of burnout patients than healthy controls during the laboratory session. After correction for age and gender the within-subjects time effect became marginally significant $(\mathrm{F}(4,160)=2.57, \mathrm{p}<0.10)$, the group effect was unaffected $(\mathrm{F}(1,40)=1.23, \mathrm{p}>0.05)$ as was the time-group interaction effect $(\mathrm{F}(4,160)=3.75, \mathrm{p}<0.05)$.

Morning cortisol levels changed over time (within-subjects time effect: $F(3,123)=28.25, \mathrm{p}<0.001)$. Burnout patients tended to have higher overall cortisol levels than healthy controls $(\mathrm{F}(1,41)=3.79, \mathrm{p}<0.10)$. No evidence for a different development of morning cortisol for the two groups emerged (time-group interaction effect: $\mathrm{F}(3,123)=1.96, \mathrm{p}>0.05)$. Post hoc analysis of the overall time effect showed significant differences between every subsequent measurement. After correction for age and gender, the time effect remained significant $(\mathrm{F}(3,117)=5.14, \mathrm{p}<0.01)$ and the group effect turned out to be also statistically significant $(\mathrm{F}(1,39)=4.87$, $\mathrm{p}<0.05)$. The time group interaction effect remained non-significant $(\mathrm{F}(3,117)=1.40, \mathrm{p}>0.05)$. Figure 5 shows that the burnout group has higher morning cortisol values during the first 30 minutes after awakening, however, one hour after awakening and at midday cortisol values of both groups have become nearly equal .

Exploratory regression analyses of associations between early morning cortisol and mental exhaustion and fatigue were carried out, using data of both groups. Positive relationships emerged between cortisol level at awakening and mental exhaustion $(\beta=0.37, \mathrm{t}=2.57, \mathrm{p}<0.05)$ and between maximal cortisol level during the first hour after awakening and mental exhaustion $(\beta=0.32, \mathrm{t}=2.20, \mathrm{p}<0.05)$. After correction for age and gender the relation between cortisol level at awakening and mental exhaustion became somewhat weaker $(\beta=0.25, \mathrm{t}=1.85, \mathrm{p}<0.10)$ as did the relation between maximal cortisol level and mental exhaustion $(\beta=$ $0.29, \mathrm{t}=2.24, \mathrm{p}<0.05)$.

Cortisol level at awakening was also positively associated with general fatigue $(\beta=0.30, t=2.06, p<0.05)$, however, the association between maximal cortisol level during the first hour after awakening and fatigue failed to reach significance $(\beta=0.22, t=1.49, p>0.05)$. After correction for age and gender, both associations became non-significant $(\beta=0.21$, $\mathrm{t}=1.50, \mathrm{p}>0.05 ; \beta=0.22, \mathrm{t}=1.54, \mathrm{p}>0.05)$.
Subjective responses during the laboratory session Psychological activation (Vigor subscale of the POMS) changed over time during the laboratory session (withinsubjects time effect: $F(4,172)=13.25, p<0.001)$. No overall difference between the groups was found $(F(1,43)=1.60, p>$ 0.05 ), nor was there any evidence for a different development of psychological activation during the laboratory session between burnout patients and healthy controls (time-group interaction effect: $F(4,172)=1.34, p>0.05)$. Post hoc analyses showed significant differences between the preparation measurement and the measurement after the speech task and between the measurement after the speech task and the first recovery measurement (irrespective of group). After correction for age and gender the time effect remained significant $(\mathrm{F}(4,164)=2.64, \mathrm{p}<0.05)$, the difference between groups became marginally significant $(F(1,41)=3.63, p<0.10)$ and the time group interaction was unaffected. The results show a tendency towards higher Vigor scores for the patient group compared to the control group and a decrease of Vigor after the stress inducing tasks for both groups.

Tension (Tension subscale of the POMS) also changed during the laboratory session (within-subjects time effect: $\mathrm{F}(4,172)=18.2, \mathrm{p}<0.001)$. Furthermore, burnout patients had overall higher tension scores than healthy controls (between-subjects group effect: $F(1,43)=26.23, p<0.001$ ) and burnout patients and healthy controls showed a different development of tension during the laboratory session (timegroup interaction effect: $F(4,172)=5.25, p<0.01)$. Post hoc analyses showed significant differences between the measurement after the speech task and the first recovery measurement and between the two recovery measurements, which could be ascribed to the patient group only. After correction for age and gender the time effect remained significant $(\mathrm{F}(4,164)=2.87$, $\mathrm{p}<0.05)$, as did the group effect $(\mathrm{F}(1,41)=14.36, \mathrm{p}<0.001)$ and the time-group interaction effect $(\mathrm{F}(4,164)=4.26$, $\mathrm{p}<0.05)$. These results indicate that the tension scores of the healthy group were relatively low and did not change during the laboratory session, while the tension scores of the patient group were considerably higher, showing a decrease after the stress inducing tasks.

\section{DISCUSSION}

The aim of the present study was to investigate differences between burnout patients and healthy controls regarding basal physiological values and physiological stress responses by examining measures of the SAM axis (HR and BP) and the HPA axis (cortisol).

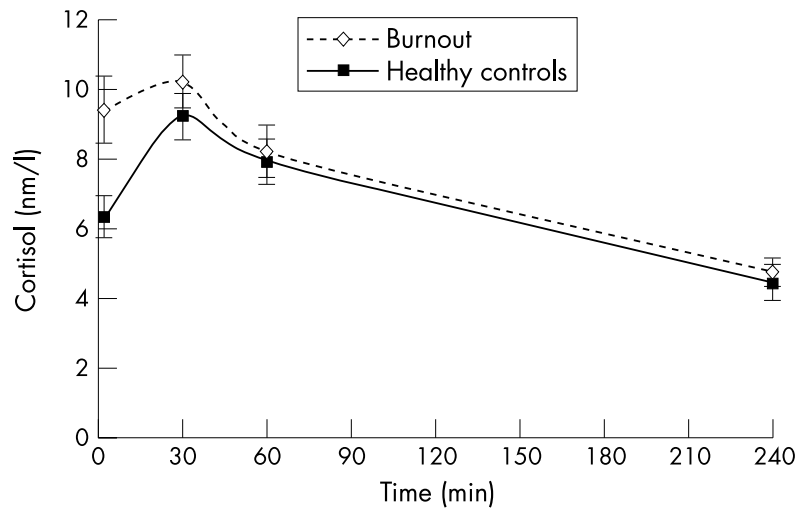

Figure 5 Mean concentration of salivary free cortisol (SEM) in burnout patients and healthy controls during the first hour after awakening and at $12.00 \mathrm{hrs}(t=1$, awakening; $t=30$, half an hour after awakening; $\dagger=60$, one hour after awakening; $t=240,12.00$ hours). Statistically significant group difference (cross-sectionally) of cortisol values at awakening is indicated by enlargement of group indicators. 
With respect to basal values of cardiovascular measures, burnout patients showed higher HR than healthy controls. These results are in line with previous studies on associations between chronic stress or workday and elevated HR..$^{15}{ }^{23}$ However, we did not find elevated BP levels as found by others. ${ }^{15-23}$ The conclusion may be that hyperactivity of the SAM axis was limited in our burnout patients. On the other hand, the lack of finding substantial differences in basal BP may also indicate that hyperactivity of the SAM axis has already recovered, as a result of the rest obtained during sick leave. This recovery could have occurred since burnout patients were tested several weeks after the chronic stressor ( such as work load or job strain) had disappeared or had been reduced. Another explanation may be that two burnout patients used anti-hypertensive medication. Post hoc analysis with exclusion of these patients did not change our findings with respect to any effect of any of the cardiovascular measures. However, average BP and HR of the patient group could have been somewhat elevated, if data of untreated hypertension of these particular patients had been at hand.

With respect to cardiovascular reactivity and recovery values during the laboratory session, differences between burnout patients and healthy controls were not obtained. Both groups show considerable cardiovascular increases during the stress inducing tasks, at least as high as the effects obtained in other studies using a laboratory stressor, ${ }^{5657}$ and recover within thirty minutes after termination of the stressor. It seems that having been exposed to chronic work stress does not extensively affect cardiovascular reactivity or recovery. Again, an alternative explanation may be that potential deviations of reactivity and recovery may have normalised within weeks after the stressor had diminished or disappeared. Also in these analyses, attention was paid to anti-hypertensive medication, and again post hoc analysis excluding these patients did not change any of the results. Our results are consistent with findings of Benschop et al, ${ }^{23}$ who found no association between cardiovascular reactivity and chronic stress, despite the fact that they obtained both elevated basal BP and HR among persons reporting chronic stress in comparison to persons not reporting chronic stress.

No differences between basal cortisol levels were obtained between burnout patients and healthy controls. These findings are partly in accordance with other findings. Steptoe et $a^{25}$ did not find any differences during the day between high and low job strain groups. Melamed et al,,$^{35}$ however, obtained higher cortisol levels at 16.00 hours in a chronic burnout group compared to a non-chronic burnout group and a group without burnout complaints. The absence of a difference in the present study could not be ascribed to relatively large inter-individual differences in measurements at 15.00 hours, as suggested by Schmidt-Reinwald et al. ${ }^{44}$

With respect to cortisol reactivity and recovery, no differences emerged between burnout patients and healthy controls. The outcomes are in accordance with results published by Roy et al, ${ }^{38}$ addressing life events and cortisol reactivity and recovery. Other studies, however, reported associations between daily stressors or job strain and vital exhaustion and cortisol reactivity, ${ }^{34} 51$ and between life stress and immunological reactivity to a laboratory stressor. ${ }^{58}$ An explanation for these inconsistent results may be that general cortisol reactivity obtained in the current study and by Roy et $a l^{38}$ was smaller than reported by others. ${ }^{44} 4659$ Consequently, group differences may remain unnoticed. A possible explanation for a somewhat smaller reactivity to the stressor, besides the use of a different cortisol assay, may be that the nature of the tasks used in the present study differed to some extent from the Trier social stress test as described by Kirschbaum et al. ${ }^{46}$ For example, a camera was used during the speech task instead of a real audience. Furthermore, the atmosphere in which the tasks were administered may have been too reassuring to evoke a substantial cortisol response, which is assumed to occur as a result of uncontrollability, inability to cope, negative affect, and (expected) negative outcomes. ${ }^{5-8}$ This suggestion is supported by relatively low tension levels reported during the tasks. However, despite the fact that Roy et $a l^{51}$ as well obtained relatively small cortisol reactivity, while also using a camera instead of a real audience during the speech task, they found an association between reactivity and daily stress. Another explanation for the relatively flat cortisol pattern during the laboratory session might be the influence of the moment of measurement. According to SchmidtReinwald et $a l^{44}$ investigating the 12 hour cortisol rhythm during daytime, cortisol concentration shows a relatively strong decrease between 13.00 hours and 15.00 hours characterised by relatively large inter-individual differences. These differences are probably largely due to variance in the consistence of the midday meal, which is known to affect cortisol concentrations. ${ }^{60}$ As a result, increases in cortisol following the stress inducing tasks might thus remain unnoticed. A flat response may, though, be considered a response, which might have shown a steeper slope without exposure to the stress test as is suggested by Roy et al. ${ }^{51}$

Burnout patients showed elevated early morning cortisol levels in comparison with healthy controls. Our findings are consistent with the studies of Melamed et al, ${ }^{35}$ Schultz et al, ${ }^{36}$ and Steptoe et $\mathrm{al}^{25}$ concerning people with high job strain, high workload or burnout symptoms and with the results of Kristenson et $a l_{,}^{34}$ concerning people reporting vital exhaustion. These findings suggest a dysregulation of the HPA axis among burnout patients. The fact that the difference seems most pronounced at the moment of awakening, burnout patients might not have recovered fully during the night, which may be a sign of "sustained activation", as is the elevated basal HR. Other authors also reported sustained activation after a working day, as indicated by elevated $\mathrm{BP}^{19}$ and adrenaline. ${ }^{61}$

It is remarkable that although burnout patients reported severe mental exhaustion, scored in the clinical range and comparable to CFS patients on general fatigue ${ }^{43}$ and reported chronic burnout complaints, they did not have blunted cortisol levels as have been found among CFS patients ${ }^{27} 28$ and PTSD patients..$^{29-31}$ On the contrary, their morning cortisol levels appeared elevated, as was found among persons reporting high job strain or high workload ${ }^{17} 25$ or vital exhaustion ${ }^{34}$ and no indication of flattening of circadian rhythm was found. In addition, the severity of mental exhaustion was positively associated with maximal early morning cortisol level. These outcomes suggest that the burnout syndrome is essentially different from CFS and PTSD. Another explanation may be that the neuroendocrine changes develop over time and thus can only be found in patients who suffer from burnout complaints for a certain long lasting period-for example, more than one year. Unfortunately, the size and distribution of the current sample did not allow us to do subgroup analysis to investigate differences in cortisol response between more and less chronic burnout patients.

The present study has several limitations. Firstly, a neuroendocrine representative of the SAM axis such as for example epinephrine was not measured. Secondly, the sample size was relatively small in comparison to similar studies using physiological measures, resulting in limited statistical power. Consequently, small effect sizes could not be detected. Thirdly, two patients used antihypertensive drugs, which may have blunted average cardiovascular activity in the patient group somewhat. Finally, the stress inducing tasks might not have caused enough distress to result in substantial HPA axis activation, thus possibly thwarting the discovery of different reactivity and recovery patterns between burnout patients and healthy controls.

In conclusion, the results of the present study suggest that there is some dysregulation of the SAM axis with respect to basal cardiovascular values, indicated by elevated HR among burnout patients. Dysregulation of the SAM axis with respect 
to reactivity and recovery values of cardiovascular measures in burnout patients was not found. In addition, deviations in HPA axis reactivity and recovery during and after stress inducing tasks were absent in burnout patients, indicating no adaptational failure. The results of current study, however, are supportive of dysregulation of the HPA axis in burnout patients as indicated by elevated early morning cortisol levels. Both elevated morning cortisol and elevated HR may be indicative of sustained activation. The outcomes of burnout patients are more similar to the results observed in persons reporting substantial job strain or vital exhaustion, than to the pattern found in patients with chronic fatigue or patients suffering from post-traumatic stress. The specific underlying mechanisms and exact meaning of these findings, however, remain to be clarified.

\section{ACKNOWLEDGEMENTS}

This study was part of the Dutch Concerted Research Action "Fatigue at work" of NWO. The Dutch Organisation of Scientific Research (NWO) and Care Research The Netherlands (ZON) funded this study. This study could not be performed without the contribution of the occupational health services AGW (Hoorn, The Netherlands) and AMD-UvA (Amsterdam, The Netherlands) and various general practitioners in and around Amsterdam. Additionally, L Geraets and F Wildschut have been of great aid during the data collection phase. The authors thank P K Beekhof for biochemical analyses and $\mathrm{W}$ Vleeming for advice.

\section{Authors' affiliations}

W De Vente, J H Kamphuis, P M G Emmelkamp, Department of Clinical Psychology, University of Amsterdam, The Netherlands M Olff, Speciality Programme Psychotrauma, Academic Medical Centre/De Meren, Department of Psychiatry, The Netherlands J G C Van Amsterdam , Laboratory of Health Effects Research, National Institute of Public Health and the Environment, The Netherlands

\section{REFERENCES}

1 Maslach C, Schaufeli, WB, Leiter MP. Job Burnout. Ann Rev Psychol 2001;52:397-422.

2 Eriksen HR, Olff M., Murison R, et al. The time dimension in stress responses: relevance for survival and health. Psychiatry Res 1999;85:39-50

3 Wessely S. Old wine in new bottles: neurasthenia and 'ME'. Psychol Med 1990;20:35-53.

4 American Psychiatric Association. Diagnostic and statistical manual of mental disorders: 4th ed. (DSM-IV), Washington: American Psychiatric Association, 1994

5 Linden W, Earle TL, Gerin W, et al. Physiological stress reactivity and recovery: conceptual siblings separated at birth? J Psychosom Res 1997;42:117-35.

6 Peters ML, Godaert GLR, Ballieux RE, et al. Cardiovascular and endocrine responses to experimental stress: effects of mental effort and controllability. Psychoneuroendocrinology 1998;23:1-17.

7 Henry JP. Biological basis of the stress response. Integrative Physiol Behav Science 1992;27:66-83.

8 Ursin H, Olff M. Psychobiology of coping and defence strategies. Neuropsychobiology 1993;28:66-71.

9 Olff $M$. Stress, depression and immunity: the role of defense and coping styles. Psychiatry Res 1999a;85:7-15.

10 Olff M, Brosschot JF, Godaert G, et al. Modulatory effects of defense and coping on stress-induced changes in endocrine and immune parameters. Int J Behav Med 1995:2:85-103.

11 Olff M, Brosschot JF, Godaert G. Coping styles and health. Pers Indiv Diff 1993;15:81-90

12 Cacioppo JT. Somatic responses to psychological stress: The reactivity hypothesis. In Sabourin M, Craik F, eds. Advances in Psychological Science, Vol. 2: Biological and Cognitive Aspects. Hove, England UK: Psychology Press/Erlbaum (UK) Taylor \& Francis, 1998:87-112

13 Frankenhaeuser $M$. The psychophysiology of workload, stress, and health: comparison between the sexes. Ann Behav Med 1991;13:197-204.

14 Dienstbier RA. Arousal and physiological toughness: implications for mental and physical health. Psychol Rev 1989;96:84-100.

15 Evans O, Steptoe A. Social support at work, heart rate, and cortisol: A self-monitoring study. J Occup Health Psychol 2001;6:361-70.

16 Goldstein IB, Shapiro D, Chicz-DeMet A, et al. Ambulatory blood pressure, heart rate, and neuroendocrine responses in women nurses during work and off work days. Psychosom Med 1999;61:387-96.
17 Steptoe A, Roy MP, Evans PO. Psychosocial influences on ambulatory blood pressure over working and nonworking days. J Psychophys 1996; 10:218-27

18 Fredrikson $M$, Tuomisto $M$, Lundberg $U$, et al. Blood pressure in healthy men and women under laboratory and naturalistic conditions. I Psychosom Res 1990:34:675-86.

19 Steptoe A, Cropley M, Joekes K. Job strain, blood pressure, and responsivity to uncontrollable stress. J Hypertens 1999;17:193-200.

20 Kawakami N, Haratani T, Araki S. Job strain and arterial blood pressure, serum cholesterol, and smoking as risk factors for coronary heart disease in Japan. Int Arch Occup Environ Health 1998:71:429-32

21 Schnall PL, Schwartz JE, Landbergis PA et al. A longitudinal study of job strain and ambulatory blood pressure: results from a three year follow-up. Psychosom Med 1998;60:697-706.

22 Tsutsumi A, Kayaba K, Tsutsumi K, et al. Association between job strain and prevalence of hypertension: a cross sectional analysis in a Japanese working population with a wide range of occupations: the Jichi Medical School cohort study. Occup Environ Med 2001;58:367-73.

23 Benschop RJ, Brosschot JF, Godaert GLR, et al. Chronic stress affects immunological but not cardiovascular responsiveness to acute psychological stress in humans. Am J Physiol 1994;266:R75-R80.

24 Steptoe A, Wardle J, Lipsey Z, et al. A longitudinal study of work load and variations in psychological well-being, cortisol, smoking, and alcohol consumption. Ann Behav Med 1998;20:84-91.

25 Steptoe A, Cropley M, Griffith J, et al. Job strain and anger expression predict early morning elevations in salivary cortisol. Psychosom Med 2000;62:286-292.

26 Frankenhaeuser $M$. A biopsychological approach to work life issues. Int J Health Serv 1989;19:747-58.

27 Demitrack MA. Neuroendocrine correlates of chronic fatigue syndrome: a brief review. J Psychiatric Res 1997;31:69-82.

28 Demitrack MA, Dale JK, Lave L, et al. Evidence for impaired activation of the hypothalamic-pituitary-adrenal axis in chronic fatigue syndrome. J Clin Endocr Metab 1991;73:1224-34.

29 Boscarino JA. Posttraumatic stress disorder, exposure to combat, and lower plasma cortisol among Vietnam veterans: findings and clinical implications. J Cons Clin Psychol 1996:64:191-201.

30 Golier J, Yehuda R. Neuroendocrine activity and memory-related impairments in posttraumatic stress disorder. Development Psychopath 1998; 10:857-69

31 Yehuda R, Southwick SM, Nussbaum G, et al. Low urinary cortisol excretion in patients with posttraumatic stress disorder. J Nerv Ment Dis 1990; 187:366-9.

32 Ockenfels MC, Porter L, Smyth J, et al. Effect of chronic stress associated with unemployment on salivary cortisol: overall cortisol levels, diurnal rhythm, and acute stress reactivity. Psychosom Med 1995;57:460-7.

33 Pruessner JC, Hellhammer DH, Kirschbaum C. Burnout, perceived stress, and cortisol responses to awakening. Psychosom Med 1999:61:197-204.

34 Kristenson M, Orth-Gomér K, Kucinskienë Z, et al. Attenuated cortisol response to a standardized stress test in Lithuanian versus Swedish men: The LiVicordia study. Int J Behav Med 1998;5:17-30.

35 Melamed S, Ugarten U, Shirom A, et al. Chronic burnout, somatic arousal and elevated salivary cortisol levels. J Psychosom Res 1999;46:591-8

36 Schultz P, Kirschbaum C, Pruessner JC, et al. Increased free cortisol secretion after awakening in chronically stressed individuals due to work overload. Stress Med 1998;14:91-7.

37 McEwen BS. Protective and damaging effects of stress mediators. New Eng J Med 1998;338:171-9.

38 Roy MP, Steptoe A, Kirschbaum C. Life events and social support as moderators of individual differences in cardiovascular and cortisol reactivity. J Personality Soc Psychol 1998;75:1273-81.

39 Pruessner JC, Gaab J, Hellhammer DH, et al. Increasing correlations between personality traits and cortisol stress responses obtained by data aggregation. Psychoneuroendocrinology 1997;22:615-25.

40 Robins LN, Wing J, Helzer J. Composite International Diagnostic Interview Manual. World Health Organization:Geneva, 1987.

41 Beck AT, Steer RA. Beck Depression Inventory Manual. New York: Harcourt Brace, 1987

42 Schaufeli W, Van Dierendock D. UBOS, Utrechtse Burnout Schaa Manual. Lisse, The Netherlands: Swets \& Zeitlinger BV, 2000

43 Bültmann U, de Vries M, Beurskens AJHM, et al. Measurement of prolonged fatigue in the working population: Determination of a cutoff point for the Checklist Individual Strength. J Occup Health Psychol 2000;5:411-16.

44 Schmidt-Reinwald A, Pruessner JC, Hellhammer I, et al. The cortisol response to awakening in relation to different challenge tests and a 12-hour cortisol rhythm. Life Sci 1999;64:1653-60.

45 Linkowski $\mathbf{P}$, Van Onderbergen A, Kerkhofs $M$, et al. Twin study of 24-h cortisol profile: Evidence for genetic control of the human circadian clock. Am J Physiol 1993;264:E173-E181.

46 Kirschbaum C, Pirke KM, Hellhammer DH. The 'Trier Social Stress Test' - a tool for investigating psychobiological stress responses in a laboratory setting. Neuropsychobiology 1993;28:76-81.

47 Olff $M$, Mulder LM, The TH, et al. The psychobiological stress response in depressed patients compared to healthy controls. Proceedings of the 29th Annual Congress of the EABCT, Dresden, 1999b:345.

48 Navazesh M. Methods for collecting saliva. Ann NY Ac Sci 1993;694:73-7.

49 Vercoulen JHMM, Alberts M, Van der Bleijenberg G. The Checklist Individual Strength. Gedragstherapie; 1999;32:131-6. 
50 McNair MD, Lorr M, Droppleman LF. Manual for the Profile of Mood States. 1971 San Diego, Educational and Industrial Testing Service.

51 Roy MP, Kirschbaum C, Steptoe A. Psychological, cardiovascular, and metabolic correlates of individual differences in cortisol stress recovery in young men. Psychoneuroendocrinology 2001;26:375-91.

52 Kirschbaum C, Kudielka BM, Gaab J, et al. Impact of gender, menstrual cycle phase, and oral contraceptives on the activity of the hypothalamus-pituitary-adrenal axis. Psychosom Med 1999;61:154-62.

53 Van Cauter E, Leproult R, Kupfer D. Effects of gender and age on the levels and circadian rhythmicity of plasma cortisol. J Clin Endocr Metab 1996:81:2468-73

54 Allen MT, Stoney CM, Owens JF, et al. Hemodynamic adjustments to laboratory stress: The influence of gender and personality. Psychosom Med 1993;55:505-17.

55 Girdler SS, Turner JR, Sherwood A, et al. Gender differences in blood pressure control during a variety of behavioral stressors. Psychosom Med 1990;52:571-591
56 Carroll D, Smith GD, Shipley M, et al. Blood pressure reactions to acute psychological stress and future blood pressure status: A 10-year follow-up of men in the Whitehall II study. Psychosom Med 2001;63:737-43.

57 Larson MR, Ader R, Moynihan JA. Heart rate, neuroendocrine, and immunological reactivity in response to an acute laboratory stressor. Psychosom Med 2001;63:493-501.

58 Brosschot J., Benschop RJ, Godaert GLR, et al. Influence of Life Stress on Immunological Reactivity to Mild Psychological Stress. Psychosom Med 1994;56:216-24

59 Kirschbaum C, Pruessner JC, Stone AA, et al. Persistent high cortisol responses to repeated psychological stress in a sub population of healthy men. Psychosom Med 1995;57:468-74.

60 Gibson EL, Checkley S, Papadopulos A, et al. Increased salivary cortisol reliably induced by a protein-rich midday meal. Psychosom Med 1999;61:214-24.

61 Sluiter JK, Van der Beek AJ, Frings-Dresen MH. Work stress and recovery measured by urinary catecholamines and cortisol excretion in long distance coach drivers. Occup Environ Med 1998;55:407-13. 